\title{
Near-Infrared Spectroscopy Correlates with Established Histological Scores in a Miniature Pig Model of Cartilage Regeneration
}

\author{
Daniel Guenther ${ }^{1}$, Chaoxu Liu ${ }^{1}$, Hauke Horstmann ${ }^{1}$, Christian Krettek ${ }^{1}$, Michael Jagodzinski ${ }^{1}$ and \\ Carl Haasper ${ }^{*}, 1,2$
}

${ }^{I}$ Trauma Department, Hannover Medical School (MHH), Hannover, Germany

${ }^{2}$ Orthopaedic Department, HELIOS-ENDO-Klinik Hamburg, Hamburg, Germany

\begin{abstract}
Near-Infrared Spectroscopy (NIRS) could be of clinical relevance in modern cartilage regeneration. In a miniature pig model correlation of measurements and histologic scores have never been used before. The data analysis was part of an animal project that investigated the effects of seeding a chondrogenic and osteogenic scaffold with a bonemarrow-derived cell concentrate and reports the histological and mechanical properties. We created 20 osteochondral defects in the femoral condyles of 10 miniature pigs. The defects were left empty (E), filled with the grafted cylinder upside down (U), or with a combined scaffold (S) containing a spongy bone cylinder covered with a collagen membrane. In the fourth group, the same scaffolds were implanted but seeded with a stem cell concentrate (S+BMCC). The animals were euthanized after 3 months, and histologic and spectrometric analyses were performed. NIRS measurements were significantly higher in the central area of the defects of group S+BMCC compared to the central area of the defects of group U. In all groups, a correlation between NIRS and the histologic scores could be demonstrated though on different levels. In the central area, a good NIRS measurement correlates with low (good) histologic scores. In group E and group $\mathrm{S}$, this negative correlation was significant $(\mathrm{p}=0.01)$. For the first time, NIRS was successfully used to evaluate osteochondral constructs in a miniature pig model.
\end{abstract}

Keywords: Cartilage, miniature pig, near-infrared spectroscopy, NIRS, osteochondral lesion, Pineda, Wakitani.

\section{INTRODUCTION}

Osteochondral defects are common in orthopedic and trauma surgery. [1] Various orthopedic procedures for osteochondral reconstruction have been developed [2] including autologous chondrocyte transplantation, osteochondral grafts [3, 4], and modern methods of regenerative medicine. Several diagnostic tools to examine the properties of reconstructions exist. Some of them require invasive procedures. NIRS has been introduced as a less invasive method for determining tissue properties in various cases. Multiple studies have shown the potential to evaluate tissues of brain [5-7], heart [8], and skin [9]. In recent years, NIRS has become established in the measurement of the properties of articular cartilage [10]. The main disadvantage of NIRS is an overlapping of the individual bands. Molecules absorb little in the visible region. In the NIR region, however, almost all molecules absorb, in particular

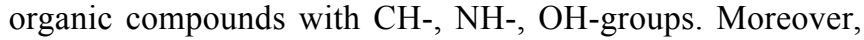
water as a main component of most organic samples, has a very broad and relatively blurred absorption peak. It is a strong NIR absorber. Using computer analysis, however, it is usually possible to compare NIR spectra with corresponding references (NIR libraries). In this way a molecular finger print is generated [11]. Osteoarthritis leads to changes in biochemical, biophysical and histological properties of the

*Address correspondence to this author at the Orthopaedic Department, HELIOS ENDO-Klinik, Hamburg, Germany; Tel: +49 403197 0; Fax: +49 403197 1966; E-mail: Carl.Haasper@helios-kliniken.de articular cartilage [12]. This leeds to a break up of the collagenglucosamine matrix and changes in water-binding properties and a shift in the ratio of the main cartilage components, water and collagen II [13]. The mechanical resistance and the biophysical characteristics of cartilage against load are changed [14]. Infrared light interacts with these cartilage components, which are affected by Osteoarthritis, such as water, water-binding properties, $\mathrm{CH}-, \mathrm{OH}-$ and $\mathrm{SH}$-groups. Studies have shown, that Osteoarthritis can be detectable with this method [15].

It was shown that the biomechanical characteristics of cartilage could be calculated from the corresponding NIRS spectra [16]. The correlation with histologic scores, like Wakitani and Pineda, has to be demonstrated. Histologic and mechanical examination protocols for the evaluation of osteochondral reconstructions have been well described in the literature [17]. The Pineda and Wakitani scores are widely used in histopathology. NIRS has been used in a rat model so far. To the authors' knowledge, it has not been used to evaluate osteochondral lesions in a miniature pig model. The aims of this study were to establish the NIRS method in a miniature pig model of modern cartilage regeneration and to evaluate the correlation of the measurements with histologic scores like Pineda and Wakitani.

\section{MATERIALS AND METHODOLOGY}

The NIRS data analysis was a part of an animal project that investigated the effects of seeding a chondrogenic and osteogenic scaffold with a bone-marrow-derived cell concentrate and reports the histological and mechanical 
properties 3 months after implantation in a miniature pig [18]. We created 20 osteochondral defects, $7 \mathrm{~mm}$ in diameter and $10 \mathrm{~mm}$ in height, in the femoral condyles of 10 miniature pigs. Autologous Cells from the iliac crest were concentrated using a concentration system (Marrowstim ${ }^{\circledR}$, Biomed, Warsaw, Indiana). $20 \mathrm{ml}$ of bone marrow aspirate were used to create $3 \mathrm{ml}$ of concentrate. The concentrate was transferred in a custom made cell seeding device (manufactured by the Institutional Central Research Laboratories of Hannover Medical School (MHH) as previously described [18]. In one group the combined scaffold (S) containing a spongy bone cylinder (Tutobone ${ }^{\circledR}$ ) covered with a collagen membrane $\left(\right.$ Chondrogide $^{\mathbb{R}}$ ) was seeded with the stem cell concentrate (S+BMCC). In the other groups, the defects were left empty (E), filled with the grafted cylinder upside down (U), or with the combined unseeded scaffold (S). The group size was $n=5$. The animals were euthanized after 3 months, and mechanical, macroscopic [18], histologic, and spectrometric analyses were performed. This publication reports the correlation of histologic and spectrometric results. Table 1 shows the allocation of pigs and condyles to each of the 4 groups. Table 2 shows the study design.

Table 1. Allocation of pigs and condyles to each of the 4 groups.

\begin{tabular}{|c|c|c|}
\hline \multirow{2}{*}{ Unilateral: Right Knee Joint } \\
\hline \multirow{2}{*}{ Animal } & \multicolumn{2}{|c|}{ Condyle } \\
\cline { 2 - 3 } & Medial & Lateral \\
\hline \hline 1 & $\mathrm{U}$ & $\mathrm{E}$ \\
\hline 2 & $\mathrm{E}$ & $\mathrm{U}$ \\
\hline 3 & $\mathrm{U}$ & $\mathrm{E}$ \\
\hline 4 & $\mathrm{~S}+\mathrm{BMCC}$ & $\mathrm{S}$ \\
\hline 5 & $\mathrm{E}$ & $\mathrm{U}$ \\
\hline 6 & $\mathrm{~S}$ & $\mathrm{~S}+\mathrm{BMCC}$ \\
\hline 7 & $\mathrm{~S}+\mathrm{BMCC}$ & $\mathrm{S}$ \\
\hline 8 & $\mathrm{~S}+\mathrm{BMCC}$ & $\mathrm{S}$ \\
\hline 9 & $\mathrm{~S}$ & $\mathrm{~S}+\mathrm{BMCC}$ \\
\hline 10 & $\mathrm{E}$ & $\mathrm{U}$ \\
\hline
\end{tabular}

Table 2. Study design.

\begin{tabular}{|c|c|c|c|}
\hline Jagodzinski et al. [18] & Study Design & \multicolumn{2}{|c|}{ Part of this Publication } \\
\hline Gait Score & & NIRS evaluation & \\
\hline $\begin{array}{l}\text { Bone marrow } \\
\text { characteristics }\end{array}$ & & $\begin{array}{l}\text { Wakitani } \\
\text { evaluation }\end{array}$ & \\
\hline Mechanical properties & & Pineda evaluation & \\
\hline ICRS evaluation & & & NIRS-Wakitani \\
\hline TRAP evaluation & & Correlation & NIRS-Pineda \\
\hline
\end{tabular}

Histological evaluation of the specimen was carried out as previously described. The modified Wakitani Score (Table 3) [19] and the modified Pineda Score (Table 4) [20] were used for evaluation of the regenerate - native cartilage interface.
Table 3. Modified Wakitani score [19].

\begin{tabular}{|l|l|}
\hline A & \\
\hline Cell morphology & 0 \\
\hline Hyaline cartilage & 1 \\
\hline Mostly hyaline cartilage & 2 \\
\hline Mostly fibrocartilage & 3 \\
\hline Mostly noncartilage & 4 \\
\hline Noncartilage only & \\
\hline B & \\
\hline Matrix staining (metachromasia) (Toludine blue-stained slides) & \\
\hline Normal (compared to host) & 1 \\
\hline Slightly reduced & 2 \\
\hline Significantly reduced & 3 \\
\hline No metachromatic stain & \\
\hline C & 2 \\
\hline Surface regularity (Total smooth area of reparative cartilage \\
compared to the whole area of the cartilage defect)
\end{tabular}

NIRS was performed using a probe connected with a personal computer as described previously [11]. The measures were performed directly on the cartilage surface after sacrificing the animals. The probe (Arthrospec, Jena, Germany) was deserved to be of similar shape and size to an arthroscopic probe with a central tip of $1 \mathrm{~mm}$. In order to measure the diffuse reflexion of cartilage, six optical fibers were integrated with a diameter of $200 \mu \mathrm{m}$ each. For spectral analysis, we used a fiber-coupled diode array microspectrometer (MCS Zeiss Micro imaging) with a spectral range of 900 to $1700 \mathrm{~nm}$ and an optical resolution of $10 \mathrm{~nm}$. A total of 50 light pulses were applied and the modified NIRS absorption was evaluated. Within $1 \mathrm{~s}$ measuring time an arthroscopy print with a value that lies between 0 and 100 appeared. This semi-quantitative value was the result of experimental studies $[14,16]$. The changes in the NIRS 
absorption correlate with the biomechanical properties (Shore hardness, Young's modulus) as well as biochemical changes (water influx and thus relative decrease of the anhydrous matrix content) and the histological changes. They reflect an integral snap-reading method of the degree of degeneration of the cartilage within the measured areas [11].

Table 4. Modified Pineda score [20].

\begin{tabular}{|l|c|}
\hline Filling of the Defect & Score \\
\hline $125 \%$ & 1 \\
\hline $100 \%$ & 0 \\
\hline $75 \%$ & 1 \\
\hline $50 \%$ & 2 \\
\hline $25 \%$ & 3 \\
\hline $0 \%$ & 4 \\
\hline Total & max. 4 \\
\hline Reconstruction of the Osteochondral Junction & Score \\
\hline Yes & 0 \\
\hline Almost & 1 \\
\hline not close & 2 \\
\hline Total & max. 2 \\
\hline Matrix Staining & Score \\
\hline Normal & 0 \\
\hline Reduced & 1 \\
\hline Significantly reduced & 2 \\
\hline Faint staining & max. 4 \\
\hline None & Score \\
\hline Total & 0 \\
\hline Cell Morphology & 1 \\
\hline Normal & 2 \\
\hline Most hyaline and fibrocartilage & 3 \\
\hline Mostly fibrocartilage & 1 \\
\hline Some fibrocartilage, but non-chondrocytic cells & 1 \\
\hline Non-chondrocytic cells only & 1 \\
\hline Total & 1 \\
\hline
\end{tabular}

Three measurements were made in the center and in the periphery of the defects and the mean values were used for further analysis. As a control, we used healthy cartilage.

If the measured values are between 0 and 40 , it is an indication for the presence of heavy degenerative damaged cartilage tissue. Values between 40 and 70 are an indication for moderate damage. Values between 70 and 100 are a predictor of little or no degeneration in the cartilage matrix [11].

The results were compared to the modified histologic Wakitani score and the histologic Pineda score and tested for correlation.
Data were processed with the statistical software package SPSS (version 20.0, SPSS Inc., Chicago, USA). Data are reported using mean values and standard deviation. Interference statistical analyses were performed for two independent samples using the Mann-Whitney U Test. The Kruskal-Wallis Test was used to check several independent samples. Spearman's rank correlation was used to characterize links between parameters. Diagrams were created with the software package Excel (version 14.2.4, Microsoft $^{\circledR}$, Redmond, USA).

The surgical procedures were approved by the local district veterinary administration and complied with the animal protection act. The study was conducted in accordance with the standards set forth in the eighth edition of Guide for the Care and Use of Laboratory Animals published by the National Academy of Sciences, The National Academies Press, Washington, DC.

\section{RESULTS}

The Wakitani Score [19] and the Pineda Score [20] were used for evaluation of the regenerate - native cartilage interface. Fig. (1) shows an example of a histological section of each group stained light green.

Figs. (2, 3) demonstrate the results of the histologic scores for the four groups.

Further histological evaluations are part of another publication [18]. We previously demonstrated in this animal model that stem cell concentrates enhance new bone formation but fail to improve mechanical properties or histological appearance of regenerated cartilage in critical size defects. Fig. (4) demonstrates the NIRS measurements of the regenerated cartilage for the four groups.

Measurements were significantly higher in the central area of the defects of group S+BMCC compared to the center of the defects of group U. Furthermore, no significant differences between the four groups could be achieved. Table 5 shows the correlation between the Wakitani Score, the Pineda Score, and the peripheral as well as central NIRS measurements in the total of all groups.

In all groups, a correlation between NIRS and the histologic scores could be demonstrated though on different levels. In the central areas, a good NIRS measurement correlated with low (good) histologic scores. In group E and group $\mathrm{S}$, this negative correlation was significant. In the central area of the defects in group $U$, no negative correlation could be demonstrated. However, the NIRS values and the histologic scores did not correlate which clearly in the peripheral areas.

\section{DISCUSSION}

Near-infrared spectroscopy is progressively establishing itself as a modern method in the assessment of defects on the articular surface. It may be particularly helpful in the evaluation of the benefit of different medical or surgical interventions in the early stages of articular cartilage damage [21]. It was demonstrated that the thickness of articular 


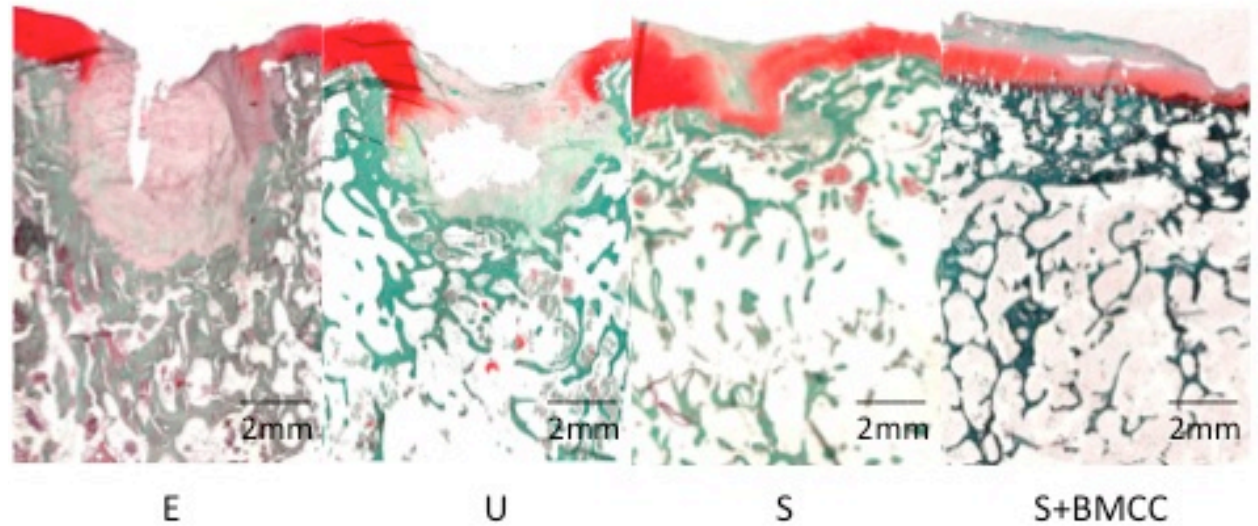

Fig. (1). Histological sections of the different groups stained light green. $E=$ empty defects, $U=$ filled with the grafted cylinder upside down, $\mathrm{S}=$ filled with a combined scaffold containing a spongy bone cylinder (Tutobone ${ }^{\mathbb{R}}$ ) covered with a collagen membrane (Chondrogide ${ }^{\mathbb{B}}$ ), $\mathrm{S}+\mathrm{BMCC}=$ combined scaffolds seeded with a stem cell concentrate.

\section{Pineda Score}

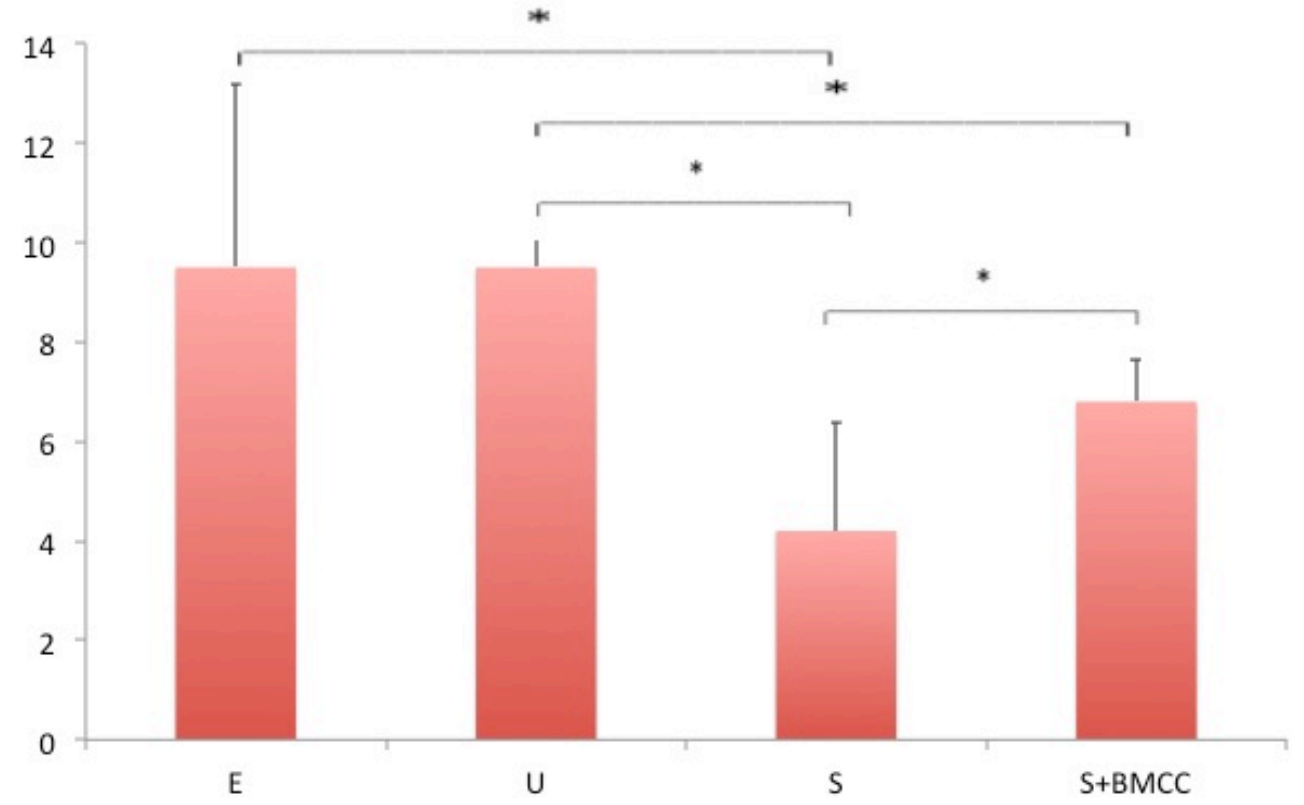

Fig. (2). Histological evaluation of the different groups $(n=5)$ using the Pineda score $(* p<0.05)$.

\section{Wakitani Score}

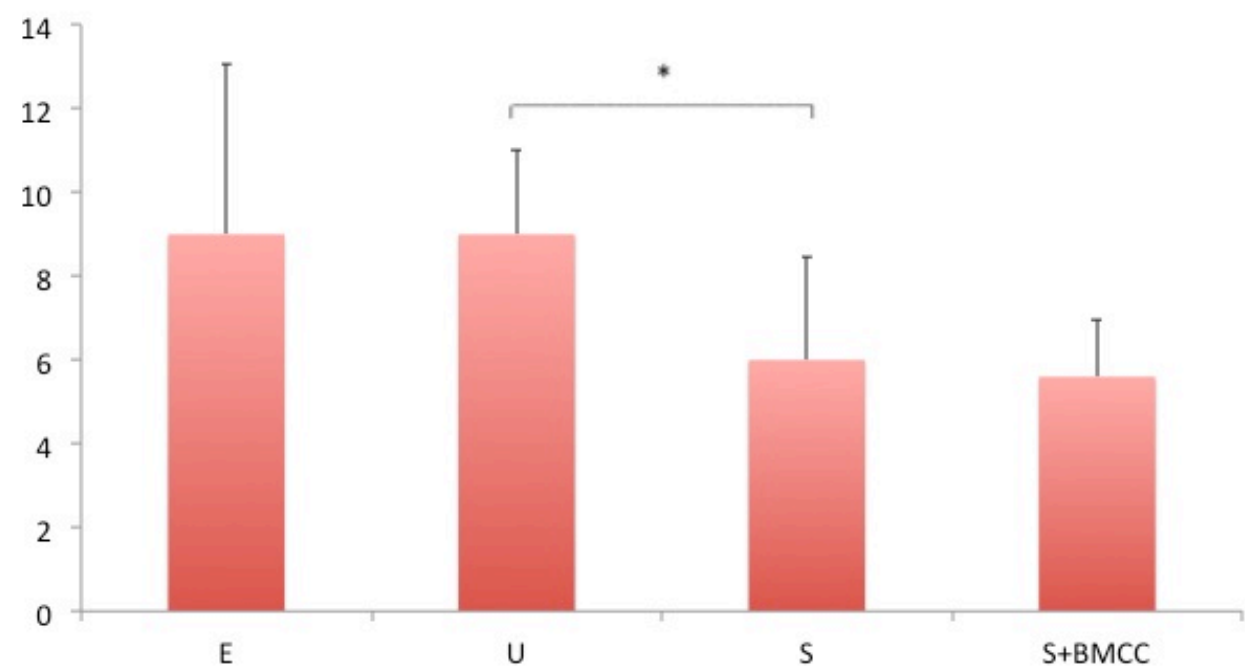

Fig. (3). Histological evaluation of the different groups $(n=5)$ using the Wakitani score $(* p<0.05)$. 


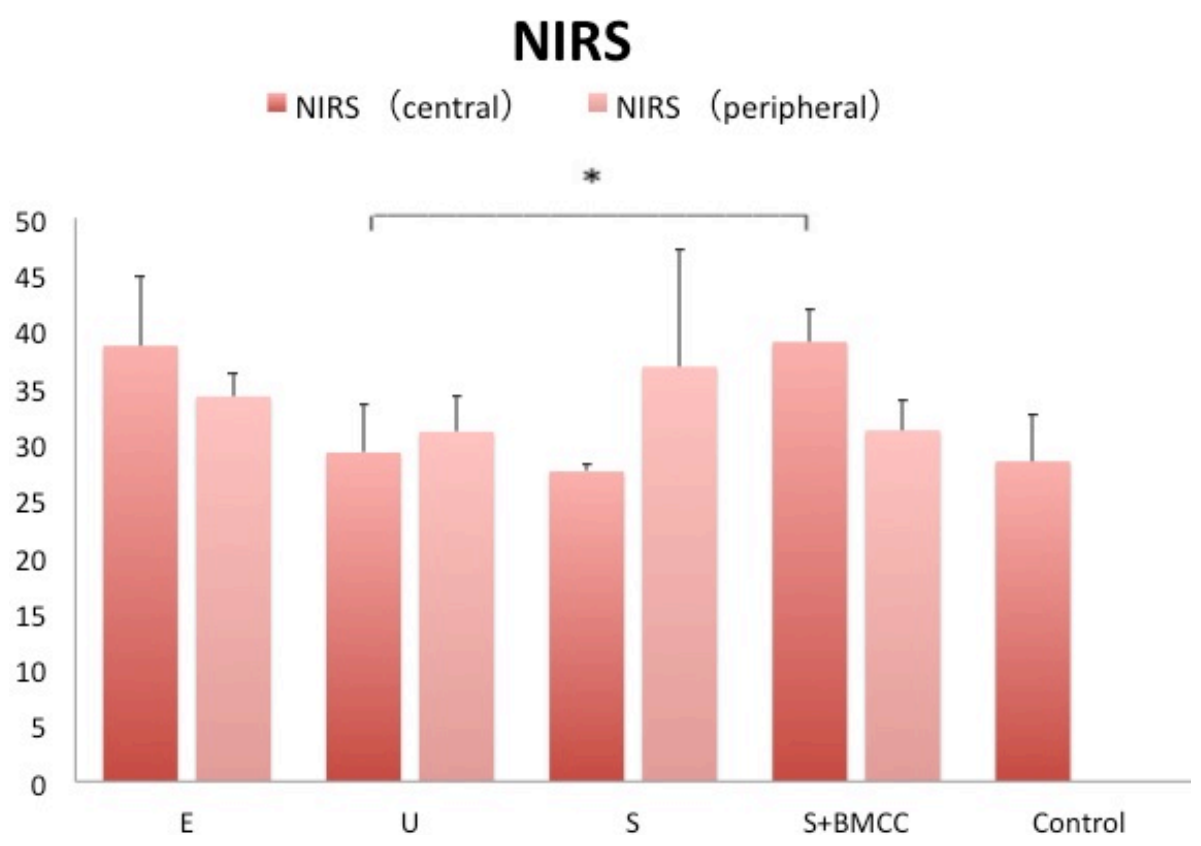

Fig. (4). NIRS measurements of the regenerated cartilage for the four groups $(n=5)(* p<0.05)$.

cartilage can be measured spectroscopically using NIR light [22]. NIRS could distinguish between normal and degraded tissue [23] and between different stages of osteoarthritic progression [24]. In arthroscopic procedures, near-infrared spectroscopy has a better inter-observer correlation than grading by ICRS score [10]. It offers the ability to monitor macromolecular content of cartilage constructs to assess engineered cartilage in situ [25]. NIRS correlates significantly with the Mankin score in an osteoarthritic rat model [26], but it has never before been established in an osteoarthritic mini-pig model to evaluate osteochondral regenerates. Furthermore, the correlation with histologic scores like Wakitani and Pineda had to be demonstrated. Studies have already shown that NIRS correlates with the ICRS-2 histological score [10]. The ICRS score of this animal project is part of another publication. To the authors' knowledge, the reisno study, that deals with the correlation of NIRS and Wakitani and Pineda scores. The ICRS score contains a surface zone score. The Wakitani score contains this as well. In the Pineda score the surface zone is not included. The penetration depth of NIR light is strongly dependant on the wave length used. The surface is always measured. That might be a reason, why the Wakitani score seems to be slightly more correlative to NIRS than the Pineda score.
Table 6 presents the correlation between the various parameters for the respective groups.

Another reason could possibly be the higher amount of sub-divisions in the Wakitani score, thus the score might be more accurate [19]. Studies have demonstrated that NIRS is a feasible tool to distinguish between normal and degraded tissue in osteoarthritis and traumatic lesions [24, 27]. The data of the NIRS measurements in group E can confirm this evidence. It could also be demonstrated that NIRS is useful to evaluate bony tissue reconstruction using scaffolds [28] as in group S. NIRS is not yet well established for cell-loaded constructs like we used in group S+BMCC. Further studies are required to evaluate spectroscopy in the tissue engineering of cartilage lesions. NIRS values and the histologic scores do not correlate clearly in the peripheral areas. The irregular reconstruction at the interface between the original cartilage and the construct might be a reason. A limitation of this study is the low number of cases due to ethical limitations in an animal project. This may be a reason for the lack of significant statements in group $U$ and group S+BMCC. Further studies with a higher case load are required. Another limitation of this study is the manual measuring which can possibly lead to overlapping of the central and peripheral measurements or of the peripheral cylinder and the flanking cartilage. However, we have to

Table 5. Correlation between the Wakitani score, the Pineda score, and the peripheral and central NIRS measurements in the total of all groups.

\begin{tabular}{|c|c|c|c|c|}
\hline & NIRS Central & NIRS Peripheral & Wakitani & Pineda \\
\hline \hline NIRS central & 1 & 0.350 & -0.379 & -0.182 \\
\hline NIRS peripheral & 0.350 & 1 & 0.004 & -0.008 \\
\hline Wakitani & -0.379 & 0.004 & 1 & $0.674^{*}$ \\
\hline Pineda & -0.182 & -0.008 & $0.674^{*}$ & 1 \\
\hline
\end{tabular}


Table 6. Correlation between the Wakitani score, the Pineda score, and the peripheral and central NIRS measurements for the respective groups.

\begin{tabular}{|c|c|c|c|c|}
\hline Group E & NIRS Central & NIRS Peripheral & Wakitani & Pineda \\
\hline NIRS central & 1 & $-1^{*}$ & $-1^{*}$ & $-1^{*}$ \\
\hline NIRS peripheral & $-1 *$ & 1 & 0.316 & 0.600 \\
\hline Wakitani & $-1^{*}$ & 0.316 & 1 & 0.949 \\
\hline Pineda & $-1^{*}$ & 0.600 & 0.949 & 1 \\
\hline \multicolumn{5}{|c|}{$* \mathrm{p}=0.01$} \\
\hline Group U & NIRS Central & NIRS Peripheral & Wakitani & Pineda \\
\hline NIRS central & 1 & -0.800 & 0.775 & 0.105 \\
\hline NIRS peripheral & -0.800 & 1 & -0.258 & 0.211 \\
\hline Wakitani & 0.775 & -0.258 & 1 & 0.544 \\
\hline Pineda & 0.105 & 0.211 & 0.544 & 1 \\
\hline Group S & NIRS Central & NIRS Peripheral & Wakitani & Pineda \\
\hline NIRS central & 1 & $-1 *$ & $-1^{*}$ & $1 *$ \\
\hline NIRS peripheral & $-1^{*}$ & 1 & -0.632 & -0.369 \\
\hline Wakitani & $-1^{*}$ & $-0,632$ & 1 & -0.028 \\
\hline Pineda & $1^{*}$ & -0.369 & -0.028 & 1 \\
\hline \multicolumn{5}{|c|}{$*_{\mathrm{p}}=0.01$} \\
\hline Group S+BMCC & NIRS Central & NIRS Peripheral & Wakitani & Pineda \\
\hline NIRS central & 1 & -0.684 & -0.725 & -0.865 \\
\hline NIRS peripheral & -0.684 & 1 & 0.725 & 0.649 \\
\hline Wakitani & -0.725 & 0.725 & 1 & 0.745 \\
\hline Pineda & -0.865 & 0.649 & 0.745 & 1 \\
\hline
\end{tabular}

mention that the diameter of the probe is only $1 \mathrm{~mm}$. Thus, a very accurate positioning is possible.

\section{CONCLUSION}

For the first time, NIRS was used to evaluate osteochondral constructs in a miniature pig model. In all groups, a correlation between NIRS and the histologic scores could be demonstrated though on different levels. In the central area of the defects a good NIRS measurement correlates with low (good) histologic scores. Further studies are required to evaluate spectroscopy in the tissue engineering of cartilage lesions.

\section{CONFLICT OF INTEREST}

The authors confirm that this article content has no conflict of interest.

\section{ACKNOWLEDGEMENTS}

Carl Haasper and Michael Jagodzinski have contributed in equal parts. We thank the team of Medimplant for the support during the animal experiments. We further wish to acknowledge the excellent support of Roland Meister. This study was supported in part by the AO Foundation's Research Fund (AO 05-H74).

\section{REFERENCES}

[1] Haasper C, Colditz M, Kirsch L, et al. A system for engineering an osteochondral construct in the shape of an articular surface: preliminary results. Annals Anat 2008; 190(4): 351-9.

[2] Bedi A, Feeley BT, Williams RJ $3^{\text {rd }}$. Management of articular cartilage defects of the knee. J Bone Joint Surg Am 2010; 92(4): 994-1009.

[3] Nazem K, Safdarian A, Fesharaki M, et al. Treatment of full thickness cartilage defects in human knees with autologous chondrocyte transplantation. J Res Med Sci 2011; 16(7): 855-61.

[4] Horas U, Pelinkovic D, Herr G, Aigner T, Schnettler R. Autologous chondrocyte implantation and osteochondral cylinder transplantation in cartilage repair of the knee joint: a prospective, comparative trial. J Bone Joint Surg Am 2003; 85-A(2): 185-92.

[5] Doi H, Nishitani S, Shinohara K. NIRS as a tool for assaying emotional function in the prefrontal cortex. Front Hum Neurosci 2013; 7: 770

[6] Torricelli A, Contini D, Pifferi A, et al. Time domain functional NIRS imaging for human brain mapping. Neuroimage 2014; 85(Pt 1): 28-50.

[7] Tsuzuki D, Dan I. Spatial registration for functional near-infrared spectroscopy: From channel position on the scalp to cortical location in individual and group analyses. Neuroimage 2014; 85(Pt 1): 92-103. 
[8] Maldonado Y, Singh S, Taylor MA. Cerebral near-infrared spectroscopy in perioperative management of left ventricular assist device and extracorporeal membrane oxygenation patients. Curr Opin Anaesthesiol 2014; 27(1): 81-8.

[9] Drakaki E, Vergou T, Dessinioti C, Stratigos AJ, Salavastru C, Antoniou C. Spectroscopic methods for the photodiagnosis of nonmelanoma skin cancer. J Biomed Opt 2013; 18(6): 061221.

[10] Spahn G, Klinger HM, Baums M, et al. Near-infrared spectroscopy for arthroscopic evaluation of cartilage lesions: Results of a blinded, prospective, inter-observer study. Am J Sports Med 2010; 38(12): 2516-21.

[11] Spahn G, Felmet G, Baumgarten G, et al. Evaluation of cartilage degeneration by near-infrared spectroscopy (NIRS): methodical description and systematic literature review. Z Orthop Unfall 2013; 151(1): 31-7.

[12] Bobinac D, Spanjol J, Zoricic S, Maric I. Changes in articular cartilage and subchondral bone histomorphometry in osteoarthritic knee joints in humans. Bone 2003; 32(3): 284-90.

[13] Buckwalter JA, Mankin HJ. Articular cartilage: tissue design and chondrocyte-matrix interactions. Instr Course Lect 1998; 47: 47786.

[14] Spahn G, Plettenberg H, Nagel H, et al. Evaluation of cartilage defects with near-infrared spectroscopy (NIR): an ex vivo study. Med Eng Phys 2008; 30(3): 285-92.

[15] Li G, Thomson M, Dicarlo E, et al. A chemometric analysis for evaluation of early-stage cartilage degradation by infrared fiberoptic probe spectroscopy. Appl Spectrosc Trial 2005; 59(12): 152733.

[16] Marticke JK, Hosselbarth A, Hoffmeier KL, et al. How do visual, spectroscopic and biomechanical changes of cartilage correlate in osteoarthritic knee joints? Clin Biomech 2010; 25(4): 332-40.

[17] Schlichting K, Schell H, Kleemann RU, et al. Influence of scaffold stiffness on subchondral bone and subsequent cartilage regeneration in an ovine model of osteochondral defect healing. Am J Sports Med 2008; 36(12): 2379-91.

[18] Jagodzinski M, Liu C, Guenther D, et al. Bone marrow-derived cell concentrates have limited effects on osteochondral reconstructions in the mini pig. Tissue Eng Part C Methods 2014; 20(3): 215-26.
[19] Wakitani S, Imoto K, Yamamoto T, Saito M, Murata N, Yoneda M. Human autologous culture expanded bone marrow mesenchymal cell transplantation for repair of cartilage defects in osteoarthritic knees. Osteoarthritis Cartilage 2002; 10(3): 199-206.

[20] Pineda S, Pollack A, Stevenson S, Goldberg V, Caplan A. A semiquantitative scale for histologic grading of articular-cartilage repair. Acta Anatomica 1992; 143(4): 335-40.

[21] Hofmann GO, Marticke J, Grossstuck R, et al. Detection and evaluation of initial cartilage pathology in man: A comparison between MRT, arthroscopy and near-infrared spectroscopy (NIR) in their relation to initial knee pain. Pathophysiology 2010; 17(1): $1-8$.

[22] Afara I, Singh S, Oloyede A. Application of near-infrared (NIR) spectroscopy for determining the thickness of articular-cartilage. Med Eng Phys 2013; 35(1): 88-95.

[23] Brown CP, Bowden JC, Rintoul L, Meder R, Oloyede A, Crawford RW. Diffuse reflectance near-infrared spectroscopy can distinguish normal from enzymatically digested cartilage. Phys Med Biol 2009; 54(18): 5579-94.

[24] Brown CP, Jayadev C, Glyn-Jones S, et al. Characterization of early stage cartilage degradation using diffuse reflectance nearinfrared spectroscopy. Phys Med Biol 2011; 56(7): 2299-307.

[25] Baykal D, Irrechukwu O, Lin PC, Fritton K, Spencer RG, Pleshko $\mathrm{N}$. Nondestructive assessment of engineered cartilage constructs using near-infrared spectroscopy. Appl Spectroscrch 2010; 64(10): 1160-6.

[26] Afara I, Prasadam I, Crawford R, Xiao Y, Oloyede A. Nondestructive evaluation of articular-cartilage defects using nearinfrared (NIR) spectroscopy in osteoarthritic rat models and its direct relation to Mankin score. Osteoarthritis Cartilage 2012; 20(11): 1367-73.

[27] Spahn G, Felmet G, Hofmann GO. Traumatic and degenerative cartilage lesions: arthroscopic differentiation using near-infrared spectroscopy (NIRS). Arch Orthop Trauma Surg 2013; 133(7): 997-1002.

[28] Cowles EA, Kovar JL, Curtis ET, Xu H, Othman SF. Near-infrared optical imaging for monitoring the regeneration of osteogenic tissue-engineered constructs. Biores Open Access 2013; 2(3): 18691. 